\title{
Parameters Affecting Shock Decision in Pediatric Automated Defibrillation
}

\author{
S Ruiz de Gauna, J Ruiz, U Irusta, E Aramendi \\ University of the Basque Country, Bilbao, Spain
}

\begin{abstract}
There are important differences between adult and pediatric ECG. To approve their use in children, the adaptation of automated external defibrillator (AED) shock advice algorithms require a specific analysis of the particular pediatric ECG characteristics.

In this study, we measured several pediatric ECG features and assessed their potential ability in the distinction between shockable and non-shockable pediatric rhythms.

For this purpose, we compiled a total of 986 pediatric ECG samples classified into four rhythm types: 540 normal sinus rhythm (NSR), 322 supraventricular tachycardia (SVT), 66 ventricular tachycardia (VT) and 58 ventricular fibrillation (VF). The samples were collected from 613 patients of mean age 7.6 years.

Five ECG parameters were calculated from the pediatric database: the pulse rate $(P R)$, the percent power around the dominant frequency $(D P)$, the percent power above $12.5 \mathrm{~Hz}(\mathrm{HP})$, the baseline content (BC) and the slope bandwidth (SB).
\end{abstract}

\section{Introduction}

Resuscitation guidelines recommend the use of automated external defibrillators (AED) in children 1-8 years of age since 2005 [1]. To approve their use in children, AED shock advice algorithms must be evaluated using pediatric rhythms, as they present differences with respect to adults. Children heart rates are higher than in adults, especially for younger children [2]. For example, most episodes of pediatric supraventricular tachycardia (SVT) exceed the typical adult threshold for a shock advice, 150 beats per minute (bpm). With adult defibrillation criteria, very prone to decide in terms of heart rate, SVT can be wrongly classified as shockable ventricular tachycardia (VT), affecting the specificity of the AED [2-4].

The differences between pediatric and adult ECG suggest a revision of the features used in adult AED algorithms in the discrimination between shockable and non-shockable rhythms and evaluate then specifically for pediatric patients. In this context, Aramendi et al compared the sensitivity of a commercial AED with a set of adult and pediatric VT samples [5]. Other studies have compared adult and pediatric ventricular arrhythmias (now including ventricular fibrillation - VF- rhythms) with respect to amplitude, frequency, complexity and regularity measurements $[6,7]$.

The present study is focused only on the analysis of pediatric rhythms. For this purpose, we have compiled a complete pediatric database, containing shockable and also non-shockable samples. We have measured five ECG parameters against our pediatric database and assessed their ability in the shock/no shock decision.

\section{Materials and methods}

\subsection{Pediatric database}

We started the pediatric database creation process in 2004. We followed the American Heart Association (AHA) guidelines to test adult AED rhythm analysis algorithms because there exist no specific recommendations for pediatric patients [8]. The first phase of the compilation was presented in 2006 [9]. Initially, we started collecting ECG samples from two Spanish hospitals, the Cruces Hospital (Barakaldo) and the La Paz Hospital (Madrid). Since then, three more Spanish hospitals have joined the project: the Donostia Hospital (San Sebastian), the San Joan de Deu Hospital (Barcelona) and the Gregorio Marañon Hospital (Madrid).

Surface ECG samples were gathered from retrospective electrophysiology studies from patients under 20 years of age. Digital recordings were obtained using the Prucka Cardiolab ${ }^{\circledR}$ and the EP-TRACER from CardioTek. Lead II was extracted and downsampled to 250 samples per second, resolution was $5 \mu \mathrm{V}$. In addition, important instances of less frequent arrhythmias were stored in paper format. Digitalization process of lead II stripes was properly described in [9] and each digitized rhythm sample was stored with a sampling frequency of $250 \mathrm{~Hz}$ and a resolution of $5 \mu \mathrm{V}$.

Three independent cardiologists classified the samples according to the different rhythm types defined by the AHA recommendations [8]. Each annotated sample was 
assigned also a shock/no shock decision. Divergences were solved after the assessment of the risks of each potential recommendation.

Following the above-mentioned criteria, we have collected more than 1100 pediatric samples with a minimum duration of $3.2 \mathrm{~s}$. For this study, we have extracted the 124 shockable registers, 66 fast VT and 58 $\mathrm{VF}$, and 862 non-shockable registers, 540 normal sinus rhythm (NSR) and 322 SVT. The samples were collected from 613 patients of mean age $7.6 \pm 4.5$ years. Further details are shown in Table 1.

Table 1. Pediatric rhythms used in the study (up to 20 years old). Age group of $\leq 8$ years is also detailed.

\begin{tabular}{|c|c|c|c|c|}
\hline \multicolumn{3}{|c|}{ Shockable rhythms } & \multirow{2}{*}{\multicolumn{2}{|c|}{$\begin{array}{c}\text { Non-shockable rhythms } \\
\text { No. }\end{array}$}} \\
\hline \multirow{2}{*}{$\begin{array}{l}\text { Age } \\
\text { group }\end{array}$} & \multicolumn{2}{|c|}{ No. } & & \\
\hline & $V F^{\mathrm{a}}$ & $V T$ & $N S R$ & $S V T$ \\
\hline$\leq 8 \mathrm{y}$ & $20 \quad(11)$ & $45 \quad(21)$ & $320 \quad(289)$ & $176 \quad(136)$ \\
\hline Total & $58 \quad(22)$ & $66 \quad(36)$ & $540 \quad(455)$ & $322 \quad(235)$ \\
\hline
\end{tabular}

${ }^{\mathrm{a}}$ The number of patients is indicated in parenthesis

\subsection{Description of the ECG parameters}

Five ECG features were calculated in signal intervals of $3.2 \mathrm{~s}$. Previously, each record was preprocessed using a band-pass filter with pass band $0.7-35 \mathrm{~Hz}$ to eliminate DC, power line interferences and base line drifts. In the following subsections a description and a graphical example of each parameter is provided.

\subsubsection{Pulse Rate}

Basic pulse rate measurements are based on the detection of QRS complexes. Typical QRS detection algorithms are often based on slope calculations, and are adequate for NSR and other sinus rhythms. However, they fail with certain type of rhythms, especially in the case of VT. In this study, the pulse rate (PR), given in $\mathrm{bpm}$, was computed using the autocorrelation of the $3.2 \mathrm{~s}$ signal window. The pulse rate is then estimated from the two dominant peaks of the autocorrelation [10]. An example of PR calculation for a NSR rhythm is shown in Fig. 1. As this method is based on the presence of certain waveform regularity it has not been applied to the VF samples.

\subsubsection{Frequency domain parameters}

For each ECG signal window, the power spectral density (PSD) was computed using the FFT algorithm with a Hanning window of $3.2 \mathrm{~s}$. Two spectral parameters were then obtained from the PSD. The first one measures the percent power in a band of $1.2 \mathrm{~Hz}$ around the dominant frequency (DF) and is denoted as DP. The DF was selected as the main PSD component in the range 0$35 \mathrm{~Hz}$. The second parameter measures the percent power above $12.5 \mathrm{~Hz}$ and is denoted as HP. When the ECG segment resembles a sinusoid, that is the case of wide complex VT, we expect high values of DP and low values of HP. In contrast, with periodic but not sinusoid rhythms (NSR and SVT), DP decreases as HP increases. Fig. 2 illustrates these calculations for a NSR and a VF sample.
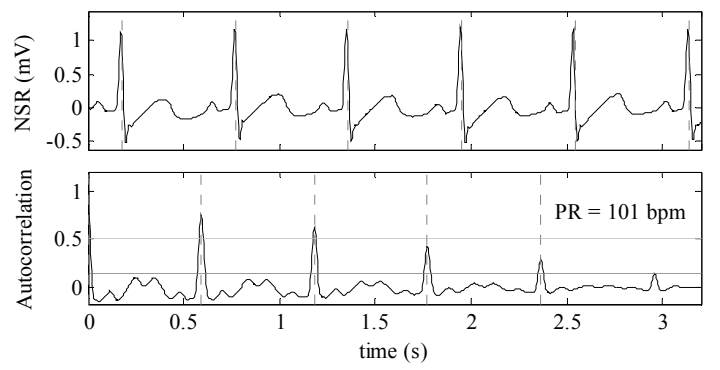

Figure 1. Calculation of parameter PR using the autocorrelation function, for a NSR rhythm.
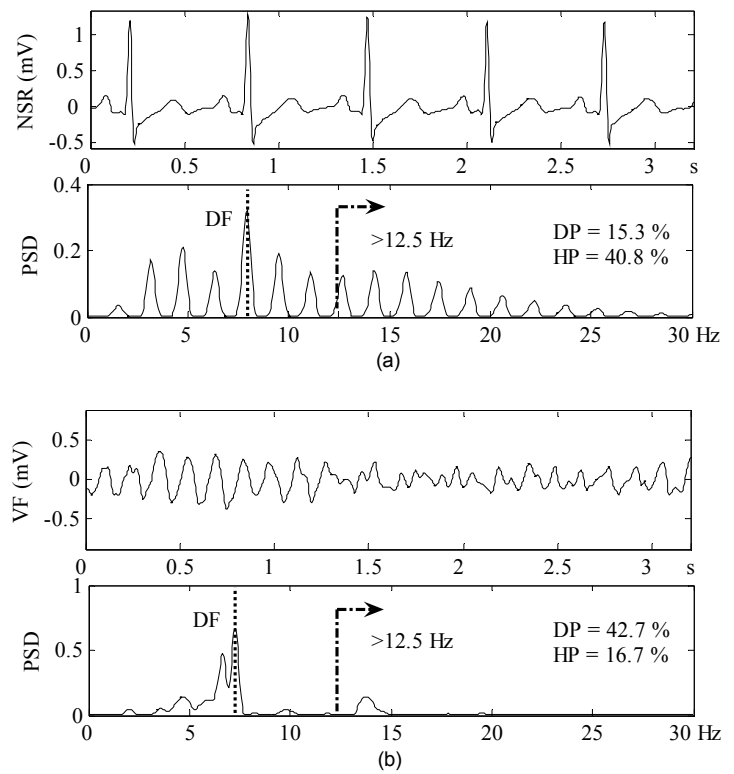

Figure 2. Calculation of the frequency parameters DP and HP for: (a) NSR, (b) VF.

\subsubsection{Time domain parameters}

The baseline content (BC) is linked to the amplitude distribution of the samples contained in each signal window. Sinus rhythms concentrate a high number of samples around the baseline, while ventricular arrhythmias present a more spread distribution of the amplitudes. First, the ECG signal window is normalized 
with respect to the maximum absolute amplitude value. Parameter $\mathrm{BC}$ is then calculated as the maximum percentage of samples in a range of $0.1 \mathrm{mV}$ around the baseline (Fig. 3).

The last parameter is based on the amplitude distribution of the normalized squared first difference of each signal window (Fig. 4). Parameter SB (slope bandwidth) is defined as the $25 \%$ percentile of the amplitude distribution. We expect lower SB values for non-shockable rhythms, due to the presence of spiky QRS complexes.
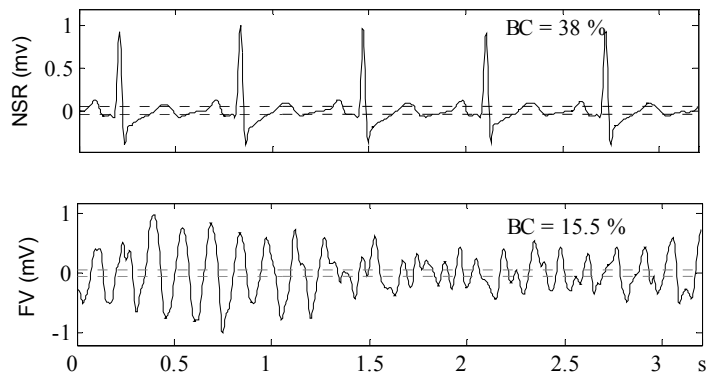

Figure 3. Calculation of parameter BC. (a) NSR, (b) VF.
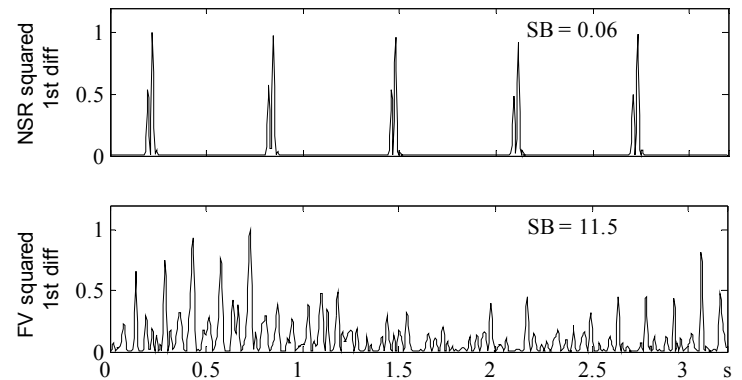

Figure 4. Normalized squared first difference for: (a) NSR, (b) VF. Rhythm examples correspond to Fig. 3.

\section{Results}

A total of 3232 windows of 3.2 s (1826 NSR, 1061 SVT, $195 \mathrm{VT}$ and $150 \mathrm{VF}$ ) were analyzed. Table 2 summarizes the results in terms of mean and standard deviation of each parameter for each rhythm type. In addition, results are presented with notched box plots in figures 5-7. Boxes whose notches do not overlap indicate that the medians of the groups differ at the $5 \%$ significance level.

Fig. 5 shows the pulse rate results. Despite of the high rates in pediatric NSR, there is a clear separation between this rhythm and the VT pulse rate. However, pediatric SVT have very high rates, the mean PR is above the adult threshold for shockable VT (150 bpm).

Fig. 6 shows the results for the two spectral parameters. DP provides a remarkable separation between the non-shock and the shock categories. Particularly interesting is the clear distance between SVT and VT, not observed from the rate measurements. On the other hand, parameter HP increases the separation between the non-shock and the shock categories. The HP values are similar among the shockable rhythm types (VF/VT) and among the non-shockable rhythm types (NSR/SVT).

Table 2. Mean (standard deviation) results for each ECG parameter and each rhythm type.

\begin{tabular}{|c|c|c|c|c|c|c|c|c|}
\hline \multirow{4}{*}{$\begin{array}{l}\text { Param. } \\
\text { PR(bpm) }\end{array}$} & \multicolumn{4}{|c|}{ Non-shockable rhythms } & \multicolumn{4}{|c|}{ Shockable rhythms } \\
\hline & \multicolumn{2}{|c|}{ NSR } & \multicolumn{2}{|c|}{$S V T$} & \multicolumn{2}{|c|}{$V T$} & \multirow{2}{*}{\multicolumn{2}{|c|}{$\begin{array}{r}\boldsymbol{V} \boldsymbol{F} \\
\mathrm{NA}^{\mathrm{a}}\end{array}$}} \\
\hline & 97 & (20) & 188 & (40) & 236 & (52) & & \\
\hline & 19.0 & $(8.1)$ & 34.0 & (14.3) & 79.8 & (9.6) & 59.6 & $(19.6)$ \\
\hline HP (\%) & 31.3 & (13.5) & 29.7 & (14.3) & 3.1 & (2.2) & 4.6 & $(5.1)$ \\
\hline BC (\%) & 35.9 & (10.4) & 21.1 & $(9.8)$ & 8.8 & (3.9) & 13.4 & (4.1) \\
\hline $\mathrm{SB}^{\mathrm{b}}$ & 0.1 & $(0.2)$ & 0.7 & $(0.1)$ & 7.9 & $(8.0)$ & 9.9 & (5.7) \\
\hline
\end{tabular}

Fig. 7 shows the results for the parameters in the time domain, $\mathrm{BC}$ and SB, respectively. From the analysis of both parameters, NSR can be clearly distinguished from the shockable VT/VF. Parameter SB also enhances the SVT separation from the shock category.

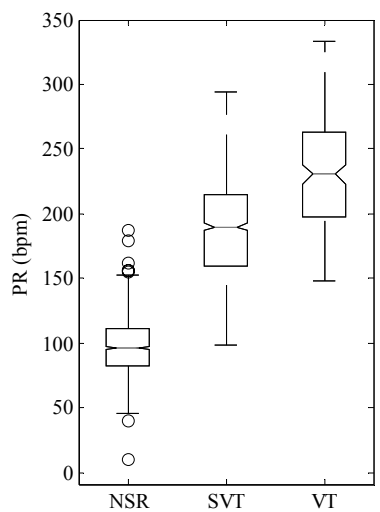

Figure 5. Box plots of the pulse rate results for NSR, SVT and VT pediatric samples.

\section{Discussion and conclusions}

Reliable AED shock advice algorithms must accurately discriminate between shockable and nonshockable rhythms. However, adaptation to pediatric patients requires a profound analysis of the different pediatric rhythms. Our objective was the analysis of four significant pediatric rhythms (NSR, SVT, VT and VF) using the information provided by five ECG parameters related to the rate, the spectral content and the morphology of the signal. 

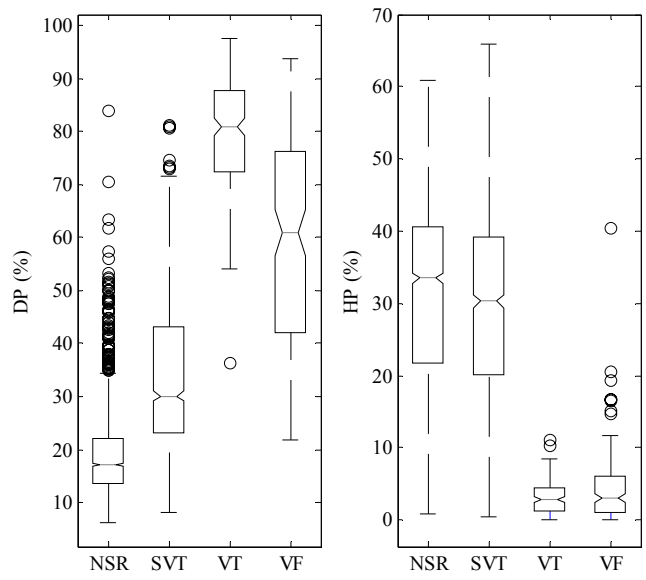

Figure 6. Box plots of the spectral parameters for: left, DP; right, HP.
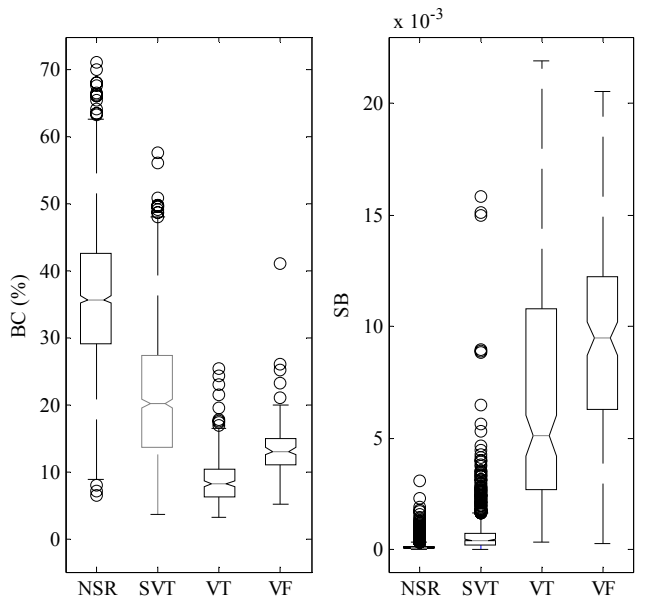

Figure 7. Box plots of parameters BC (left) and SB (right).

In particular, parameter PR reveals a strong overlap between SVT and VT rates, due to the higher pediatric SVT rate. The two spectral parameters, DP and HP seem adequate for the distinction between SVT and VT, sometimes borderline even for electrophysiology experts. Mean (standard deviation) results for parameter DP were 34.0 (14.3) for SVT and 79.8 (9.6) for VT. Results for parameter HP were 29.7 (14.3) for SVT and 3.1 (2.2) for VT. Finally, the time domain parameters, BC and SB, should be considered in the distinction between sinus and ventricular rhythms.

We think that the conclusions derived from this study are valuable for a future adaptation of adult shock advice algorithms to pediatric patients.

\section{Acknowledgements}

The pediatric database creation process was financially supported by Osatu S. Coop. (Ermua, Spain) and the Spanish Ministry of Education and Science.

\section{References}

[1] Biarent D, Bingham R, Richmond S, et al. European Resuscitation Council Guidelines for Resuscitation 2005 Section 6. Paediatric life support. Resuscitation 2005;67(1):S98.

[2] Atkins LA, Scott WA, Blaufox AD et al. Sensitivity and specificity of an automated defibrillator algorithm designed for pediatric patients. Resuscitation 2008;76:16874.

[3] Cecchin F, Jorgenson D, Berul C, et al. Is Arrhythmia Detection by Automatic External Defibrillator Accurate for Children? Circulation 2001;103:2483-8.

[4] Atkinson E, Mikysa B, Conway J, et al. Specificity and sensitivity of automated external defibrillator rhythm analysis in infants and children. Ann Emerg Med 2003;42(2):185-96.

[5] Aramendi E, Irusta U, Ruiz de Gauna S, Ruiz J. Comparative Analysis of the Parameters Affecting AED Rhythm Analysis Algorithm Applied to Pediatric and Adult Ventricular Tachycardia. Computers in Cardiology 2007;34:419-422.

[6] Irusta U, Ruiz J, Ruiz de Gauna S, Aramendi E. Sequential VT/VF Discrimination Algorithm Based on Wave Mode Sample Entropy for Adult and Pediatric Patients. Computers in Cardiology 2007;34:229-232.

[7] Irusta U, Ruiz J, Aramendi E, Ruiz de Gauna S. Amplitude, frequency and complexity features in paediatric and adult ventricular fibrillation. Resuscitation 2008:77;S53-S53.

[8] Kerber R, Becker L, Bourland J, et al. Automatic external defibrillators for public access defibrillation: Recommendations for specifying and reporting arrhythmia analysis algorithm performance, incorporating new waveforms, and enhancing safety. Circulation 1997;95:1677-82.

[9] Irusta U, Aramendi E, Ruiz de Gauna S, Ruiz J, Gutierrez JJ, Bodegas A, Pastor E, Benito F. Development of a Pediatric ECG Rhythm Database for the Assessment of the Rhythm Analysis Algorithms of Automated External Defibrillators. Computers in Cardiology 2006;33:609-612.

[10] Ruiz J, Aramendi E, Lazkano A, Leturiondo LA, Almaraz S, inventors; Método para la determinación de la frecuencia de forma de onda de una señal ECG. Spain patent 2232223. 2006.

Address for correspondence

Sofia Ruiz de Gauna

School of Engineering

Electronics and Telecommunications Department

Alameda Urquijo, s/n, 48013-Bilbao (Spain)

sofia.ruizdegauna@ehu.es 